\title{
MULTI-ORBIT SYNCHROTRON WITH FFAG FOCUSING FOR ACCELERATION OF HIGH INTENSITY HADRON BEAMS
}

\author{
R.Ueno, M.Matoba, Kyusyu Univ., Fukuoka, Japan \\ T.Adachi, M.Fujieda, S.Ishi, K.Koba, S.Machida, Y.Mori, R.Muramatsu, C.Ohmori, I. Sakai, \\ Y.Sato,T.Uesugi, K.Umezawa, Y.Yamamoto, M.Yoshii, $\quad$ KEK-Tanashi, Tokyo, Japan \\ K.Noda, M.Kanazawa, S.Yamada, NIRS, Chiba, Japan
}

\begin{abstract}
Multi-orbit synchrotron(MOS) with fixed field alternating gradient(FFAG) focusing is attractive for acceleration of high intensity hadron beams because acceleration cycle could be increased. The magnetic field of MOS is static, therefore, the repetition rate of acceleration could be increased more than 10 times larger than that of ordinary rapid cycling synchrotron(RCS) if an efficient high voltage RF accelerating system becomes available. Recently, a new type of high gradient RF cavity (HGC) using high permeability magnetic alloy (MA) has been developed and MOS with FFAG focusing becomes very promising. In order to clarify the feasibility of rapid cycling MOS (RCMOS) experimentally, proof-of-principle (POP) machine, which accelerates protons up to $1 \mathrm{MeV}$ with $1 \mathrm{kHz}$ repetition, is under development. We have also made several designs on high intensity proton accelerators with RCMOS for various applications such as accelerator driven system(ADS) for energy breeder, spallation neutron source and proton driver for muon collider.
\end{abstract}

\section{INTRODUCTION}

High intensity medium energy ( $1 \mathrm{GeV} \sim 10 \mathrm{GeV}$ ) proton beams are required for many applications such as spallation neutron source, accelerator-driven system (ADS) for nuclear energy production, proton-driver for muon collider, etc. In these applications, large beam power of more than $1 \mathrm{MW}$ is requested. In order to realize such large beam power with ordinary proton synchrotron, rapid cycling of beam acceleration is inevitably. For example, an high intensity cyclotron with superconducting magnets has been discussed for ADS as a possible candidate for the cyclic accelerators. This is believed mostly because of the experience at the PSI cyclotron, which has obtained about more than 1MW beams so far. As for a synchrotron, it has been thought that it would be almost useless for ADS because the operation is a pulsed mode and the average beam current is small. The magnetic field is time varying according to beam acceleration in the synchrotron, the eddy-current power loss in the magnets becomes serious when the repetition rate of the accelerating cycle is increased and the magnetic field ramping exceeds more than 200T/sec. On the other hand, the accelerated particle number per pulse is limited by the space-charge effect. Practically, the maximum repetition of the rapid cycling synchrotron is limited *E-mail:Yoshiharu.Mori@kek.jp to be less than $50 \mathrm{~Hz}$ or so. Therefore, the maximum available beam power would be at most about 1MW[1]. However, the beam in the synchrotron is stable, because it is strongly focused in the transverse and longitudinal directions, and the instantaneous beam current in the ring becomes very large. Fixed-field alternating gradient (FFAG) synchrotron, thus, becomes attractive for this purpose.

Another issue is an electric power efficiency in operation of high intensity accelerator. For such large beam power accelerator, the electric power required for operation increases to an acceptable level if the electric power efficiency is small. The operational electric power efficiency of the accelerator is defined by the ratio of the total beam power to the total electric power requested for operation of the whole accelerator system. In order to make the ADS, for example, in a realistic manner, the electrical-power efficiency should be at least more than $30 \%$. The beam power can be expressed by the product of the beam energy and the averaged beam current. The requested beam power for ADS would be at least $10 \mathrm{MW}$. Since a beam energy of $1-3 \mathrm{GeV}$ is most practical for ADS if the accelerated particles are protons, the average beam current should be about $10 \mathrm{~mA}$. The accelerator comprises mainly the magnet and the rf accelerating systems. During operation, the $80-90 \%$ of the total electricity of the accelerator is dissipated for these two systems. The electric power consumed by the magnet system can be dramatically reduced by using a superconducting technique, and can become negligibly small. On the other hand, the electric power dissipated by the rf accelerating system would still be an issue even if a superconducting rf system is applied. The rf electric power for the accelerating cavity system is given by,

$$
P=\frac{V^{2}}{R_{S} L} .
$$

Here, $\mathrm{V}$ is the total rf voltage requested for beam acceleration, Rs the effective shunt impedance of the accelerating cavity per unit length and $\mathrm{L}$ the total length of the accelerator. In a linear accelerator, the total length of the accelerator should be kept small because of the site limitation and also to minimize the initial construction cost. Thus, a superconducting $\mathrm{rf}$ cavity system is inevitably essential in a linear accelerator system to reduce the total $\mathrm{rf}$ power requested for operation by increasing the effective shunt impedance. On the other hand, for a cyclic accelerator, such as cyclotron or synchrotron, the situation is more reluctant, 
because the cyclic accelerator is regarded as being a very long accelerator. More than $50 \%$ electric power efficiency seems to be possible in a cyclic accelerator, even if the normal conducting rf cavity is used.

\section{MULTI-ORBIT SYNCHROTRON WITH FFAG FOCUSING}

A multi-orbit synchrotron(MOS) using fixed-field alternating gradinet(FFAG) focusing seems to be very attractive for this purpose, because the repetition rate of the accelerating cycle could be raised ten times or more compared to that of the ordinary synchrotron. The idea of a MOS using a FFAG was proposed independently by Ohkawa[2], Symon[3] and Kolomensky[4] in the early 1950's, and electron-beam machines demonstrating this principle have been successfully built in the MURA project.[3] In MOS with FFAG focusing, where the magnetic field is constant in time, the shape of the magnetic field should be such that the betatron tunes for both the horizontal and vertical planes should be constant for all closed orbit, and departing from all of the dangerous resonance lines. The condition above is called "zero-chromaticity".

$$
\left.\frac{\partial}{\partial p}\left(\frac{K}{K_{0}}\right)\right|_{\vartheta=\text { const. }}=0,\left.\quad \frac{\partial n}{\partial p}\right|_{\vartheta=\text { const. }}=0 .
$$

A magnetic field satisfying the scaling conditions described above must generally have the form ,

$$
B(r, \theta)=B_{i}\left(\frac{r_{i}}{r}\right)^{n} F\left(\theta-\varsigma \ln \frac{r}{r_{i}}\right),
$$

Table.1 Fundamental parameters of $1.5 \mathrm{GeV}$ FFAG synchrotron.

$\begin{array}{ll}\text { Injection Energy } & 0.25 \mathrm{GeV} \\ \text { Extraction Energy } & 1.5 \mathrm{GeV} \\ \text { Beam Intensity } & 5.5 \times 10^{13} \mathrm{ppp} \\ \text { Repetition Rate } & 750 \mathrm{~Hz} \\ \text { Average Beam Current } & 6.6 \mathrm{~mA} \\ \text { No. of Sectors } & 16 \\ \text { Circumference Factor } & 2.68 \\ \text { Average Beam Radius } & \\ \quad \text { injection } & 12.2 \mathrm{~m} \\ \quad \text { extraction } & 13.4 \mathrm{~m} \\ \text { Magnetic Field } & \\ \quad \text { injection } & 0.536 \mathrm{~T} \\ \quad \text { extraction } & 1.5 \mathrm{~T} \\ \text { Field Index } & 10.5 \\ \text { Effective Field Index } & 3.9 \\ \text { Spiral Angle } & 64.6 \mathrm{deg} \\ \text { Fractional Angle } & 8.34 \mathrm{deg} \\ \text { Betatron oscillation tune } & \\ \quad \text { horizontal } & 3.73 \\ \quad \text { vertical } & 3.23 \\ \text { Transition Gamma } & 3.442 \\ \text { Max. RF Voltage } & 0.56 \mathrm{MV} \\ \text { RF Frequency } & 2.39 \mathrm{MHz} 3.3 \mathrm{MHz}\end{array}$

where $\varsigma$ is a spiral angle. If $\zeta$ is zero, the magnetic field does not depend on $\theta$, and the corresponding orbit points are distributed on a radial vector. The type of having this magnetic shape is called "radial sector". One the other hand, if $\theta$ behaves in a logarithmic manner, such as

$$
\theta-\varsigma \ln \frac{r}{r_{i}}=\text { const. }
$$

the orbits remain geometrically similar, but move around the beam center towards larger radii. This type is called " spiral sector".

The MOS with FFAG focusing is very attractive for acceleration intense proton beams as described above and several proposals have been submited.[5][6] However, no practical proton-beam machine has been built so far. One of the most difficult technical issues to realize a high-repetition MOS is rf acceleration. The requested accelerating rf voltage per one turn is

$$
\Delta V=2 \pi(1+n)\left(\frac{d r}{d t}\right) p .
$$

Here, $d r / d t$ is the orbit excursion rate. In the case of a $1 \mathrm{GeV}$ MOS with the repetition rate of $1 \mathrm{kHz}$, the requested rf voltage becomes almost $1 \mathrm{MV}$. This is a rather difficult number if an ordinary ferrite-loaded rf cavity is applied, which

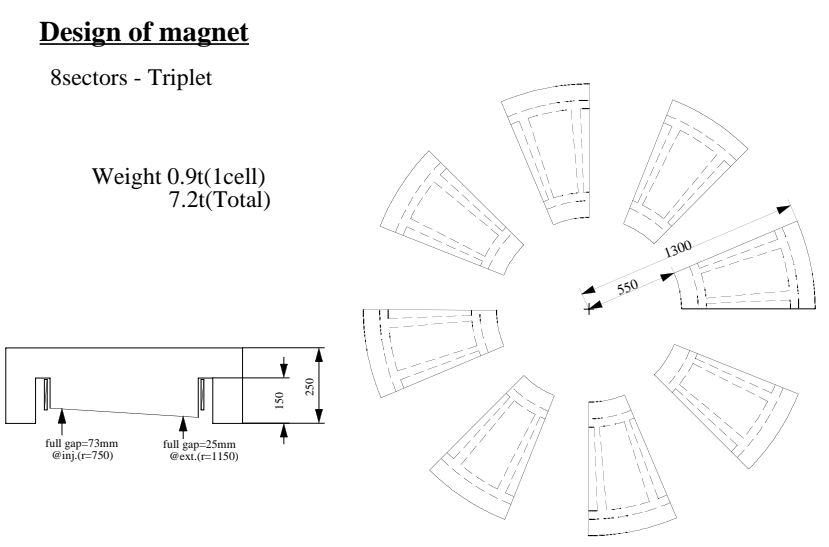

Fig.1 $1 \mathrm{MeV}$ MOS proof-of-principle machine.

has been conventionally used for the proton synchrotron so far. In the ordinary ferrite-loaded rf cavity, the maximum accelerating field gradient is at most $10 \mathrm{kV} / \mathrm{m}$ or so. Therefore, more than $100 \mathrm{~m}$ long straight sections are necessary for the rf cavities in the ring, although the total circumference of the $1 \mathrm{GeV}$ MOS would be less than $150 \mathrm{~m}$. Recently, a new type of high-gradient rf cavity using a highpermeability magnetic alloy has been developed at KEK for the JHF project, and a field gradient of $100 \mathrm{kV} / \mathrm{m}$ has been successfully achieved. [7] Using this high-gradient cavity, the most difficult technical issue in realizing a highrepetition MOS can be solved.

A preliminary design of the $1.5 \mathrm{GeV}$ and $10 \mathrm{MW}$ beam power MOS with FFAG focusing has been carried out.[8] The fundamental parameters are listed in Table 1. 


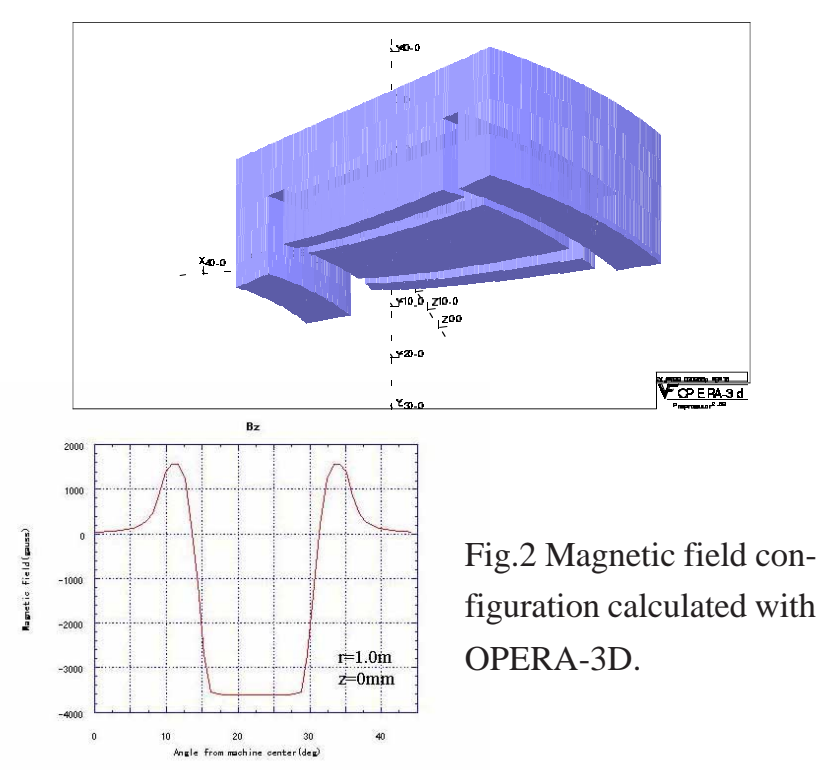

\section{1-MeV AND 1-kHz POP MACHINE}

In order to clarify the availability of very rapid cycling in MOS with FFAG focusing, we have been developing a small POP( proof-of-principle) machine. In this POP machine, the maximum energy is limited to $1 \mathrm{MeV}$ but the repetition rate of acceleration is $1 \mathrm{kHz}$. The magnet configuration is a radial sector type and eight fold symmetry is chosen as shown in Fig.1. Each sector consists of three dipole magnets which form a triplet focusing configuration DFD(defocus-focus-defocus) and field index of each dipole magnet is 2.5 , respectively. The maximum magnetic fields of the focusing and defocusing dipole magnets are $0.5 \mathrm{~T}$ and $0.2 \mathrm{~T}$, respectively. The magnetic field configurations in three dimensional directions are calculated with OPERA3D (Fig. 2) and their results are used for beam tracking simulation. The average beam radius changes from $0.81 \mathrm{~m}$ to $1.13 \mathrm{~m}$ according to the increase of beam energy from $100 \mathrm{keV}$ to $1.1 \mathrm{MeV}$. The half gap heights of the magnet at the radius of $0.75 \mathrm{~m}$ and $1.15 \mathrm{~m}$ are $73 \mathrm{~mm}$ and $25 \mathrm{~mm}$, respectively.

The betatron tunes for horizontal and vertical directions are varied with field index and the product of the magnetic field and the effective magnet length (B1-product). The lines in Fig. 3 show the variations of the betatron tunes for both directions calculated with the SAD code. The design values of betatron tunes for horizontal and vertical directions are 2.25 and 1.35 , respectively. The open squares and circles in Fig. 3 show the betatron tunes for two differ-

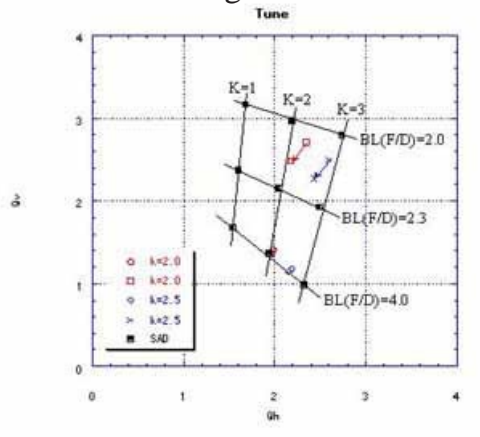

Fig. 3 Betarton tune diagra of $1 \mathrm{MeV}$ POP machine.. ent field indices of 2.5 and 2.0, respectively, which are obtained with beam tracking simulation for 3D magnetic field configurations presented in Fig. 2. The betatron tunes are slightly different from those estimated by SAD and change gradually during acceleration. The beam behaviors in the transverse direction obtained by beam tracking simulation are shown in Fig. 4. The rf frequency changes from
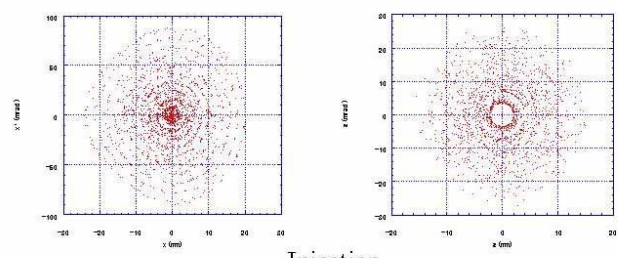
Injection

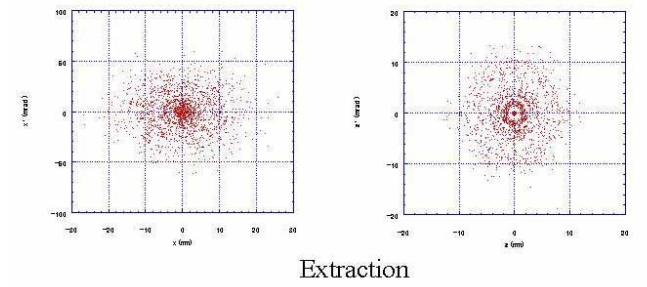

Fig. 4 Particle tracking simulation in transeverse direction.

$0.85 \mathrm{MHz}$ to $2.05 \mathrm{MHz}$. At the condition of the constant radial displacement as a function of time $(\mathrm{dr} / \mathrm{dt}=$ const.), the rf voltage has to be increased from $1.1 \mathrm{kV}$ to $3.1 \mathrm{kV}$. This rf voltage can be easily obtained by a magnetic alloy(MA) loaded rf cavity.[7] The longitudinal beam motions calculated by beam tracking simulation are shown in Fig.5.
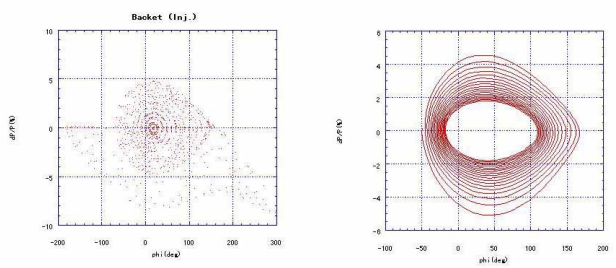

Fig.5 Particle tracking simulation in longitudinal direction.

\section{SUMMARY}

A $1.5 \mathrm{GeV}$ and $10 \mathrm{MW}$ multi-orbit proton synchrotron (MOS) with fixed field alternating gradient (FFAG) focusing has been designed. Although the repetition rate for accelerating cycle is rather high, $750 \mathrm{~Hz}$, the required rf voltage is relatively small, only 580 , because of its small ring size. A $1 \mathrm{MeV} \mathrm{POP}$ (proof-of-principle) proton machine with $1 \mathrm{kHz}$ repetition is under development.

\section{REFERENCES}

[1] JHF Accelerator Design Study Report, KEK Report 97-16.

[2] T.Ohkawa; Proc. of annual meeting of JPS (1953).

[3] K.R.Symon et al.;Phys. Rev. 103 (1956) 1837.

[4] A.A.Kolomensky et al.;ZhETF 33,298(1957).

[5] R.L.Kustom et al.;IEEE,NS-32,2672(1985).

[6] H.Sasaki;GEMINI Design Report, KEK-Report (1985).

[7] Y. Mori, Proc. of EPAC98, Stockholm, 1998, page 299.

[8] Y.Mori, Proc. of Thorium Fuel Cycle, Genshikaku Kenkyu, 43(1999)27. 